\title{
The Impact of Authority on Cooperation: A Cross-Cultural Examination of Systemic Trust
}

\author{
P. Mitkidis • D. Xygalatas • N. Buttrick • \\ M. Porubanova $\cdot$ P. Lienard
}

Received: 4 August 2014 / Revised: 27 September 2014 / Accepted: 30 September 2014 /

Published online: 7 October 2014

(C) Springer International Publishing 2014

\begin{abstract}
In this article, we examine the effects of authority on systemic trust in four different countries (the Czech Republic, Denmark, Mauritius, and the USA). We used a modified Trust Game to assess whether information about salient authority either in a religious or in a secular domain has the effect of enhancing trust in situations where social information is limited. We found that patterns of behavior differ by country, with the USA and the Czech Republic behaving similarly with relatively high trust for secular authorities, medium trust for religious authorities, and low trust for nonauthorities, and that Denmark and Mauritius behave similarly, with medium trust for secular authorities, high trust for religious authorities and low trust for non-authorities. We discuss possible explanations involving how people use social information to make decisions in situations of uncertainty.
\end{abstract}

Keywords Trust $\cdot$ Authority $\cdot$ Religion $\cdot$ Secular $\cdot$ Cross-cultural economic experiment

P. Mitkidis · N. Buttrick

Center for Advanced Hindsight, Social Science Research Institute, Duke University, Durham, USA

P. Mitkidis · D. Xygalatas

Interacting Minds Centre, Department of Culture and Society, Aarhus University, Aarhus, Denmark

P. Mitkidis $(\bowtie)$

Interdisciplinary Centre for Organizational Architecture, Aarhus University, Aarhus, Denmark

e-mail: mitkidispan@gmail.com

D. Xygalatas

Department of Anthropology, University of Connecticut, Storrs, USA

D. Xygalatas

Laboratory for the Experimental Research of Religion, Masaryk University, Brno, Czech Republic

M. Porubanova

Department of Psychology, State University of New York at Farmingdale, Farmingdale, USA

P. Lienard $(\bowtie)$

Department of Anthropology, University of Nevada, Las Vegas, USA

e-mail: pierre.lienard@unlv.edu 


\section{Introduction}

Everyday interactions in modern state societies yield particular social situations which pose many thorny challenges to human decision-making. A citizen must regularly engage, randomly or systematically, with strangers about whom she lacks knowledge of specific abilities, motivations and personality or character traits, and with whom further engagement will probably not be required. Social agents are also typically specialized in very distinct fields of activity, which makes it hard to rely on one's own experience when interacting with others. However, many ordinary interactions do not consist in situations of social exchange involving mutual trust between individuals hence they do not require much more than good coordination between self-interested social agents. In organically structured and efficiently functioning complex societies, the coordination of anonymous agents, allowing for the spontaneous emergence of social order, is mainly achieved via institutions, social, political and economic, and norms, laws and regulations (e.g. Granovetter 2005; Weber 1947).

In situations of social exchange where cooperation occurs, the level of trust determines the outcome of the exchange. Much of our economic life involves such social exchanges. A properly functioning and trusted basic legal framework allows for the enforcement of such things as, contractual arrangements, or consumer, employer and employee protection and reduces the uncertainty rising from the potential of conflict between individual and collective interests to tolerable levels. But life in a complex state society also creates many situations which are not governed by institutional or legal norms, situations which could potentially lead to beneficial cooperative social exchanges between individuals who do not know each other. In such situations there is great uncertainty about other agents, given the non-existence of a previous history of interaction. In the absence of a regulatory framework of some sort, the anonymity and specialization that complex state societies naturally create may make cooperation more difficult, even if all interacting parties perceive the potential benefits of an exchange. The more pronounced the anonymity, the wider the gap between areas of specialization and the greater the lack of common knowledge, the more likely that trust will become the primary issue that agents willing to cooperate will face.

This article is concerned with such informal exchange situations, in which individuals have to make decisions to cooperate in conditions of increased uncertainty, and when mutual trust is hard to achieve given that other's motivations, dispositions and incentives are either impossible or prohibitively costly to acquire. More specifically, we ask whether providing information about expertise and domain authority of agents - on whose decision the outcome of a cooperative interaction depends - leads systematically to increased trust, coaxing agents to take greater risk. We will see that, for specific experts and authorities, we observe inferences of stereotypical attributes determining cooperative behavior in an unrelated domain of application (i.e. expertise-unrelated economic exchange). We then offer hypotheses for why that might be the case.

\section{Background}

For the purpose of our research, we adopt a relational understanding of trust, following Hardin (2002). We opt for a definition of trust as encapsulated interest, covering 
situations in which agent $\mathrm{A}$, the truster, trusts another agent $(\mathrm{B})$, the trustee, because $\mathrm{A}$ perceives (possibly erroneously) that B's interest encapsulates her own (Hardin 2002). There is an important difference between Hardin's concept of trust and what could be called "reliability". Hardin's trust goes beyond the simple alignment of incentives or interests and implies that part of A's representation of the situation of social exchange includes a form of evaluation of B's commitment to fulfill A's trust, that is, a representation of B's purported trustworthiness (Hardin 2002). The encapsulated interest can be minimal and very much tied to a specific modality of interaction (i.e. relations between bartering partners) or can be extensive, involving, for instance, sustained reciprocity (i.e. cooperation among friends) (Hardin 2002).

Gambetta (1988) explains what it means for a social agent (A) to trust another (B): agent $\mathrm{A}$ assesses the probability of B behaving in a beneficial (or neutral) way to her as being high enough as to motivate her to cooperate. Trust and its associated expectations allay some of the concerns that one's partner might decide to act opportunistically (Gambetta 1988). But opportunistic behavior must be possible if trust is to be involved. Indeed, trust prods agents into making riskier bets on the success of cooperation, where available evidence alone would not suffice to fully justify one's engagement (Lewis and Weigert 1985). Thus, in such conditions, the agent's decision to cooperate depends heavily on the evaluation of the other agent's incentives, motivations and abilities that determine the latter's behavior. According to Cook, Hardin, and Levi (2005: 7-8) that assessment can be based either on the understanding of the structure of interaction, inherent constraints, risks, and benefits (e.g. reputational) of the interaction, or the personal knowledge of the other agent.

Focusing on the psychological dimensions of trust, it has been firmly established that people vary in their general disposition to trust and to be trustworthy; in their responsiveness to trusting behavior; and in their susceptibility to guilt and risk aversion as incentives for favorable response to gestures enabling cooperation (Gurtman 1992; Sorrentino et al. 1995; McCabe et al. 2003; Eckel and Wilson 2004; Cox 2004; Charness and Dufwenberg 2006; Bacharach et al. 2007; Sapienza et al. 2007; Schechter 2007; Houser et al. 2010). When explaining dispositional trust, Rotter (1971, 1980) posits that people evolve generic beliefs about other people's overall dispositions from early and successive trust-related experiences. People develop a stable, generalized and diffuse default disposition for trust based on the succession of individual occurrences of social exchange situations (Rotter 1980). Although an interesting perspective, we do not pursue such line of research but we focus on what we would call systemic trust, that is, trust rooted in the properties of social and cultural systems and not strictly in knowledge about the personality of the interacting agents (Butler 1991).

Research on trust, social categories and gender helps to illustrate the role that knowledge about the category membership of individuals has on trust. For example, women, as members of a well-identified category, are generally more trusted to cooperate than males in prisoner's dilemma games by all participants, even without exhibiting more trustworthy behavior. (Orbell et al. 1994). The difference in general expectations about male and female behavior seemed to have been based on a stereotypical gender-based-category evaluation bias (Kramer 1999; Orbell et al. 1994). Orbell et al. also found that when it came to predicting how cooperative a particular participant would be, neither male nor female participants relied on gender stereotypes (Orbell et al. 1994). Strong categorical expectations generally bias people's 
expectations about others and can be helpful at first in information-poor conditions. However, the stereotypical expectations do not systematically replace expectations about particular individuals in novel circumstances. In Orbell et al.'s experiment, target-based expectations seem to readily override category-based expectations (Orbell et al. 1994). The shift between stereotypical and individual-specific expectations is probably linked to the informational constraints inherent to each particular situation of social exchange. Our experimental manipulation drastically constrains the amount of personal and social information available. No reference to salient-category membership is provided at any time but for information about specific positions and roles in organizational structures, which brings us to the other relevant point that Kramer makes about another category of trust.

In addition to trust based on category membership, trust can also be based on an agents' role within society. Role-based trust rests upon the knowledge of the typical properties, characteristics, requirements, duties and responsibilities of salient positions in particular organizations (Kramer 1999). Such knowledge may help to foster trust in people occupying such positions, while reducing the need for costly acquisition of the more personal information necessary to calibrate an agent's interaction with potential cooperators (Kramer 1999). Kramer therefore sees that in specific conditions, roles can become "proxies" from which to infer more essential characteristics of particular organization members: "[as long as] role occupancy signals both [...] intent [on the part of an agent] to fulfill [her] obligations and competence in carrying them out, individuals can rely on a presumption that the agent will be trustworthy, even in the absence of personalized knowledge or prior history of interaction" (Kramer 1999: 578). Thus, general knowledge about barriers and costs to entry into organizational roles, along with the perception of the efficacy of accountability and compliance mechanisms within the organization sustains role-based trust (Kramer 1999: 578). We selected our representatives of expert categories with salient authority accordingly.

Both category-based and role-based trusts are underlain by trust in the social system as a whole. Systemic trust, as we conceptualize it in the present work, rests to a great extent on how much confidence individuals have in stable organizations, norms, and institutions and their sustained effects on people's behavior, and facilitates the stability of social systems and organizational structures. An agent's systemic assessment of the trustworthiness of organizational authorities is based on broad and largely prototypical evaluations of organizational hierarchies (Simmel 1950; Parsons 1951; Luhman 1979; Fukuyama 1995; Aghion and Tirole 1997; Lane 1998). General organization-specific trustworthiness assessments can be very helpful in information-poor social conditions, such as is often likely to occur in our modern social world. Our experimental manipulation evaluates whether stereotypical knowledge about recognized expertise and authority impacts people's cooperative behavior in situations where social information about interacting agents is reduced to a minimum. Systemic trust assessments are also necessarily grounded in personal and collective evaluations of authorities, generated through the cumulative experience of particular interactions with actual representatives of such social categories (Shamir and Lapidot 2003). Therefore, systemic trust says something about the actual structure of trust in public entities. Our experimental manipulation will allow us to advance some hypotheses about the reasons behind the privileged trust allocation to specific public entities in the different countries where we ran the experiment. 
Thus, apart from perceived personal characteristics, traits, dispositions and concerns, trust-warranting properties of social agents can be inferred from the existing structure of interactions. Adapting Zucker's model (1986) to our purpose, we identify several major systemic ways for trust to emerge: through recurrent transactions in habitual processes of impersonal exchange; through institutional designs establishing structures of more or less informal incentives, disincentives and rewards (given the complexity of our societies note that many modern-day spheres of activity are not covered by such institutional elaborations); and through the perception of category- and role-based social characteristics, features and conditions. In this paper, we do not investigate general or personal trust but focus only on the consequences on trust of organizational structures and of the knowledge that social agents have of those structures.

\section{The Choice of the Countries, the Experts, the Authorities and the Non-Authorities}

Our experimental design is targeted at whether information about salient expertise (with authority either in religious or in secular domains) has an effect on cooperation when social information is lacking. We ran the experiment cross-culturally in four different countries: the Czech Republic (CZ), Denmark (DK), Mauritius (MU) and the USA (US). The four countries were selected to evaluate how much of the trust-enhancing effect of information about authority and expertise is associated to particular cultural systems and how much of it is an associated property of the type of highly diversified social landscapes we observe in state and nation-state environments.

The Economist in its 2013 Democracy Index ranked the four countries among the 25 full democracies found around the world (Democracy Index, The Economist Intelligence Unit 2013). Full democracies are countries in which political and civil liberties are fully guaranteed, due process is systematically followed, and the political and mass communication cultures greatly promote social and political participation (Democracy Index, The Economist Intelligence Unit Limited 2013: 28). All four countries are socially stratified, though none is strictly hierarchical (i.e. social mobility is possible and does occur). Although all of them are strongly centralized, the four countries show evidence of powerfully marked corporate groups (ethnic, political, social and religious) and of strong expression of individualism observable in many modern democratic states. None of those four countries have authoritarian regimes. The indices presented below help to build a quantitative image of the particular features (democracy, individualism, ethnic and religious diversity) of the four selected countries.

The CZ, MU, and the US are ranked 17th, 18th and 21st in the Democracy Index and they have respective overall Democracy Index scores of 8.19/10, 8.17/10 and 8.11/ 10. The score is an aggregate of several distinct category scores: (1) electoral process and pluralism, (2) civil liberties, (3) functioning of government, and (3) political participation and political culture. Most indices for those three countries are clustered around similar high values, but for the lower political participation in MU (5/10, compared to $6.67 / 10$ for the CZ and $7.22 / 10$ for US) and for the lower score in the category of civil liberties for the US (8.53/10, compared to 9.71 for MU and 9.41 for the CZ). DK does not belong to the CZ-MU-US cluster. It is ranked 4th, with an overall Democracy Index of 9.52/10 and high scores in the categories electoral process and pluralism (10/10), functioning of government (9.64/10) and political participation 
$(8.89 / 10)$, and all indices higher than in the other three selected countries. It is relevant to insist on the general Democracy Index scores of two of our selected countries. Both $\mathrm{MU}$ and the $\mathrm{CZ}$ are among the countries with a high Democracy Index (compared to a world average of 5.52/10). Either MU or CZ align with the performances of their regional surroundings (Africa or Central Europe). MU is the single African country considered a full democracy, while the $\mathrm{CZ}$ scores much higher in the Democracy Index score than the other countries of Central Europe. ${ }^{1}$ (Democracy Index, The Economist Intelligence Unit 2013)

Three of the four countries, the CZ, DK and the US have a moderate score in the Religious Diversity Index developed by the Pew Research Center. The Religious Index is computed based on the percentage of each country's population belonging to eight major religious groups: Buddhists, Christians, Folk Religions Hindus, Jews, Muslims, Other Religions, Unaffiliated (Pew Research Center 2014). CZ scores 4.1/10, DK scores 3.3/10 (European average of 4.6/10), and US scores 4.1/10 (North American average of 4.2/10) (Pew Research Center 2014). MU is the exception with a high score of 6.7/10 (though the African average is 5.8/10) (Pew Research Center 2014). The Ethnic Fractionalization Index, developed by (Alesina et al. 2003), is another interesting index that can help us to categorize the four countries we have selected. The least ethnically diverse of the four countries is DK with a score of .0819, then the CZ with .3222, MU with .4634 and then US with .4901 . For comparison purposes, in 2003, Uganda had the highest Fractionalization Index of the world with .93 (Alesina et al. 2003). Note that once again DK is somewhat isolated from the cluster formed by the other three countries.

Overall, all the selected countries share similar characteristics: similar political systems, good political representation and participation, high Democracy Index scores, average to high Religious Diversity Index scores, and moderate Ethnic Fractionalization scores. The selected countries have very different dominant cultural groups: Anglo-Saxon in the US, Slavic in the CZ, Afro- and Asian-Mauritian in MU, and Northern European in DK. It is relevant to note already here that the greater differences between several indices appear between DK and MU. The two countries differ significantly in their social and cultural homogeneity, with MU having to deal with greater ethnicity-based socio-political and economic divisions (Carroll and Carroll 2000; Greig et al. 2011; Kasenally 2011; Richards 2007; Srebrnik 2000).

For the expert and authoritative roles we test, we have selected a variety of representatives of common professions and religious denominations found around the world (see table 1, left column). ${ }^{2}$ We chose experts and authorities based on their social visibility, level of rigorous and lengthy training, and presence or knowledge of the trade

\footnotetext{
${ }^{1}$ Most central European countries are considered flawed democracies, that is, countries that have a Democracy Index score below 8.00 out of 10. Slovenia, Slovakia, Poland and Hungary, for instance, are ranked respectively 28th (DI score: 7.88/10), 40th (7.35/10), 44th (7.12/10) and 49th (6.96/10).

${ }^{2}$ The procedure for selecting the professions to be used in the experimental protocol in each one of our countries was as follows: We first established an extensive list of various professions. In each country we then asked four local informants independently which professions of the list they can easily recognize. All the professions were ranked in order of ease of recognition, from the most recognizable to the least one. Then the informants were asked to categorize all the professions of the list into either one of the three categories: secular authority, religious authority and non-authority. The selection and ranking of the informants drove our adoption of the country-specific lists of professions to be used in the experiment. When there was the possibility to enhance the correspondence of the lists between countries, we did so.
} 
Table 1 List of stimuli

\begin{tabular}{ll}
\hline Experts and authorities & Non-authorities \\
\hline Monk (Religious authority) & Alternative Healer \\
Baptist minister (Religious authority) & Artist \\
Buddhist monk (Religious authority) & Bus/Tram driver \\
Imam (Religious authority) & Butcher \\
Nurse (Secular authority) & Farmer \\
Medical doctor (Secular authority) & Florist \\
Pastor in danish folk church (Religious authority) & Gardener \\
Pastor in mainstream protestantism (Religious authority) & Grocery store keeper \\
Priest in roman catholic church (Religious authority) & Janitor \\
Priest in orthodox church (Religious authority) & Kiosk keeper \\
Priest in hinduism (Religious authority) & Receptionist \\
Rabbi (Religious authority) & Secretary \\
Teacher (Secular authority) & Student \\
University professor (Secular authority) & Taxi driver \\
Temple president (Secular authority) & Cashier in supermarket \\
Judge (Secular authority) & \\
\hline
\end{tabular}

in the respective societies in which our experiment was run. Furthermore, the selected experts and authorities had to be susceptible to loss of reputation, gossip or social retaliation were they to fail or not perform at the level required by their specialization. All non-authorities are specialists that, although providing necessary services, are typically not granted much social recognition (see table 01, right column). As for the experts and authorities, non-authorities too can be exposed to gossip (e.g. unscrupulous florists, careless bus drivers, cunning cab drivers) and social retaliation (skirting the florist shops, taking cabs, taking buses).

\section{Participant Pools and Recruitment}

Participants were 112 (mean age: 27.48, SD: 11, 60 females): 37 for US (mean age: 39.08, SD: 13,07, 15 females), 20 for DK (mean age: 22.15, SD: 3, 20 females), 21 for MU (mean age: 21.33, SD: 1.93, 7 females) and 34 for the CZ (mean age: 22.12, SD: 1.38, 19 females). The DK, CZ, and MU participants were recruited through the use of fliers among university student populations. All participants in DK were native Danish. All participants in $\mathrm{CZ}$ were Eastern European, with a majority native CZ. The majority of the MU participants were Asian Mauritians (Mauritian Hindus). The U.S. participants were recruited via an advertising board at a State Fair in Durham, North Carolina. The U.S. participants were mainly residents of North Carolina. The majority of US participants were white, with black or African-American participants as the largest minority. All participants gave written informed consent prior to inclusion in the study. Participants were compensated and fully debriefed at the conclusion of the study. Each participant received either $\$ 10$ (in the US), 100 DKK $(\approx \$ 18$-in DK), 200 MUR $(\approx \$ 7$ - 
in $\mathrm{MU})$, or $100 \mathrm{CZK}(\approx \$ 6$-in the $\mathrm{CZ})$. All amounts in local currencies correspond to a similar purchasing power in their respective country.

\section{Methods, Material and Procedure}

We used a modified Trust Game (TG - Berg et al. 1995), with a within (treatment) and between (countries) subjects design. This game is typically played as follows. A first participant (A) is asked to decide what portion of an initial endowment she wishes to invest, by sending it to another participant (B). Participant A and B do not know each other and are not allowed to converse otherwise. Before participant A finalizes her investment decision, $\mathrm{A}$ and $\mathrm{B}$ are informed that the dollar amount invested is to be tripled before reaching B. B then has then to decide what portion of the whole amount she wants to keep, and what portion to send back to participant A. Given the incentive structure of the game, the Nash equilibrium predicts that in conditions of full information, participant A should skirt investing any money because of the risk of defection on the part of B. In laboratory settings though, participants do not seem to follow the game-theoretic solution and are commonly observed to invest and to send back fair amounts of money. Berg et al. (1995) found that most participants invest or send back about $50 \%$ of the amount they receive either as original endowment or from the first participants. Despite various modifications of the incentive structure of the game, such results are robust, and people reliably give more than predicted by the Nash equilibrium (Johnson and Mislin 2011).

In our modified design, we only have one participant (A), who, unbeknownst to her, is playing with a computerized participant B. Participant A enters the lab room where the experimenter and a computer monitor are found. The experimenter tells the participant that she is about to play a game and in doing so she will interact with other players via the computer. The participant is also told that she will have to decide whether and how much of her original monetary endowment to invest in her partner, and that she will have to estimate what each one of the other participants will send back to her. The experimenter then proceeds to launch the program (SuperLab software 4.5, Cedrus, Phoenix, Arizona), and to ask the participant to read the cover story and the instructions (fig. 1). The experimenter then leaves the participant alone in the experimental room. The participant goes through $5 \mathrm{TG}$ training trials to ascertain that the instructions and the logic of the game have been properly understood.

The experimental design involves matching the participant with imaginary agents having professions fitting 1 of 3 conditions: (1) Secular Authority (SA), (2) Religious Authority (RA) and (3) Non-Authority (NA). Each of these conditions is presented to the participant as a different profession. For example, the participant could be told that she is about to interact with a medical doctor (considered here a Secular Authority). To create a realistic situation, the participant would be instructed that the (putative) game partner (our computer-simulated medical doctor) will introduce him/herself via a computer message (see Fig 1 - Cover Story). The participant is also told that the name of her game partner will appear blanked using a series of question marks, to preserve the partner's anonymity (in effect blocking any reference to gender identity). The participant is informed that after that introduction she will have to decide whether 


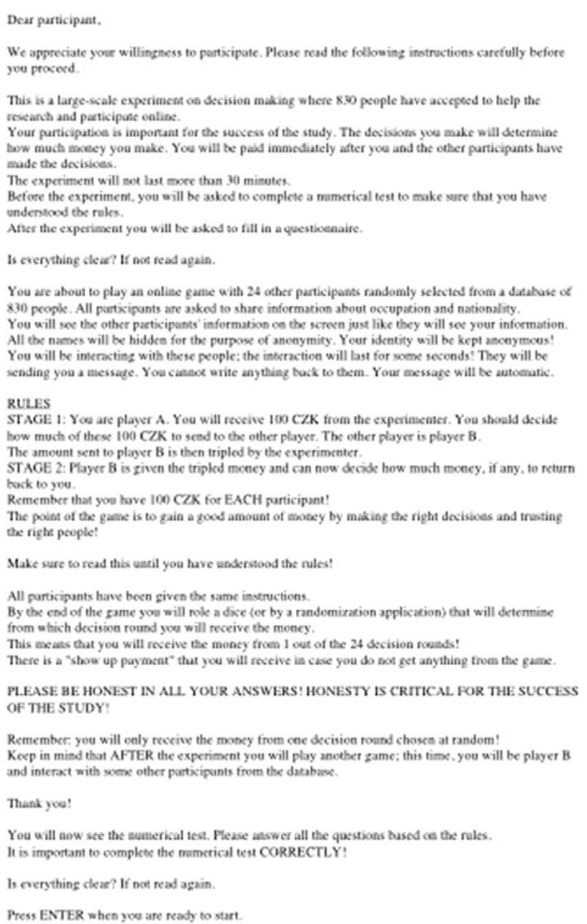

Fig. 1 Cover story (left side), instructions (left side) and stimuli example (right side)

she wishes to send money from her original endowment to the doctor and if so how much (Investment measurement - Fig. 1). The participant is informed that the amount she sends will be multiplied by three and that the doctor will then decide what portion of the new amount is to be returned to the participant. The participant is then asked what amount she thinks the doctor will send back (Expectation Measurement). Each participant interacted in a random sequence with a succession of pre-programmed agents, each belonging to one of the categories of experts or non-experts mentioned above. Each participant interacted with representatives of all three categories (SA, RA, and NA). Additionally, the participant is told that after finishing her roles as player A, she will play the game again, but this time as player B. This instruction was part of the cover story only, to make the situation realistic, and never took place. The participant only played as player A, never as player B.

In the experimental phase, participants went through 4 blocks of 6 trials (24 decisions in total), with a filler task presented after every six decisions (a video someone shooting a basketball, during which the participant had to estimate whether each successive shot would be successful or not). Trials were presented randomly, and participants were able to participate anonymously. Participants were informed at the beginning of the procedure that their final payment would be decided by the outcome of a randomly-selected trial, decided via the roll of a 24-sided die at the end of the experiment. The amount of money that was supposedly sent back by the putative player in that trial would be the participant's final compensation. We did so to preserve participant incentives across all trials. 
At the completion of the experiment, participants answered a short questionnaire on socio-demographic characteristics (gender, age) and questions related to religiosity, authoritarian personality, and education level. All instructions, tasks and questionnaires were translated from English to each of the specific country's vernacular language and then back-translated to English. Any discrepancy between translations was then addressed and resolved. The experiment lasted about 20 min per participant.

\section{Results}

We ran several statistical tests to analyze the effect of information about expertise and authority on trust in the TG in the four different countries. We first report statistics from the repeated-measures analysis with the two basic measures, namely Investment and Expectation, as distinct measures. As both measures of Investment and Expectation show similar effects, we combine them together as a measure of Trust and use that aggregate measure for all further statistical analyses (see also correlations between the different measures, Fig. 2). We then report how authority (secular and religious)/nonauthority impacts behavior in each country individually. Finally, we conduct post-hoc repeated-measures analysis and repeated contrasts analysis by splitting religious authority into in-group and out-group (country-specific splitting ${ }^{3}$ ) to explore if this alters the observed effects.

Repeated-measures ANOVAs, with Investment and Expectation as different variables, revealed a highly significant interaction effect between the different types of authority (SA, RA, NA) and both measures of Investment $F(6,212)=6.818, p=.000$, $\eta_{\mathrm{p}}{ }^{2}=.039$ and Expectation $F(6,212)=2.960, p=.009, \eta_{\mathrm{p}}{ }^{2}=.023$. When collapsing the measures of Investment and Expectation into a single aggregate measure of Trust, a repeated measures ANOVA revealed a highly significant interaction between the different types of Authority (SA, RA, NA) and the measure of Trust $F(6,212)=$ 5.332, $p=.000, \eta_{\mathrm{p}}{ }^{2}=.035$ (all three medium $\eta_{\mathrm{p}}{ }^{2}$; Cohen 1988).

In order to investigate the interaction effect, we split the analysis by the factor country (Fig. 3), which reveals an interesting pattern: the US and the CZ behave in one pattern, while MU and DK behave in a separate pattern, especially for trust in RA (Fig. 3): US: $F(2,72)=7.788, p=.001, \eta_{\mathrm{p}}{ }^{2}=.088, \mathrm{CZ}: F(2,64)=11.1897, p=.000$, $\eta_{\mathrm{p}}{ }^{2}=.172$, MU: $F(2,38)=13.129, p=.000, \eta_{\mathrm{p}}{ }^{2}=.240$, and DK: $F(2,38)=32.390$, $p=.000, \eta_{\mathrm{p}}{ }^{2}=.428$.

Based on the above results, we ran simple post-hoc tests, to explore how each country differs from the others. In the Trust measure for SA there are no significant differences (all $p>.341$ ). In the Trust measure for RA, US significantly differs from DK $(p=.012)$ and the $\mathrm{CZ}$ differs from both MU $(p=.023)$ and DK $(p=.002)$. In the Trust measure for NA there are no significant differences between countries (all $p>.121$ ).

In order to explore more systematically the effect of RA, we focused singly on that measure and split RA into in-group RA and out-group RA, based on the distinct

\footnotetext{
${ }^{3}$ Examples of the specific religious in-group vs. out-group stimuli per country:

US religious in-group: Monk, Baptist Minister, Protestant Pastor vs. out-group: Imam, Rabbi

CZ religious in-group: Monk, Priest in Roman Catholic Church vs. out-group: Imam, Rabbi

MU religious in-group: Monk, Priest in Hinduism, vs. out-group: Rabbi, Buddhist Monk

DK religious in-group: Monk, Pastor in Danish Folk Church vs. out-group: Imam, Rabbi
} 


\begin{tabular}{|c|c|c|c|c|c|c|c|c|c|c|}
\hline \multicolumn{11}{|c|}{ Correlations } \\
\hline & $\begin{array}{c}\text { SA } \\
\text { Expectation }\end{array}$ & SA Trust & $\begin{array}{c}\text { RA } \\
\text { Investment }\end{array}$ & $\begin{array}{c}\text { RA } \\
\text { Expectation }\end{array}$ & RA Trust & $\begin{array}{c}\text { NA } \\
\text { Investment }\end{array}$ & $\begin{array}{c}\text { NA } \\
\text { Expectation }\end{array}$ & NA Trust & $\begin{array}{c}\text { RA in-group } \\
\text { Trust }\end{array}$ & $\begin{array}{c}\text { RA out- } \\
\text { group Trust }\end{array}$ \\
\hline \multirow[t]{3}{*}{ SA Investment } & .681 & .919 & .580 & .362 & .499 & .776 & .651 & .763 & .458 & .401 \\
\hline & .000 & .000 & .000 & .000 & .000 & .000 & .000 & .000 & .000 & .000 \\
\hline & 112 & 112 & 111 & 112 & 111 & 112 & 111 & 111 & 112 & 111 \\
\hline \multirow[t]{3}{*}{ SA Expectation } & & .914 & .441 & .435 & .465 & .487 & .721 & .629 & .431 & .339 \\
\hline & & .000 & .000 & .000 & .000 & .000 & .000 & .000 & .000 & .000 \\
\hline & & 112 & 111 & 112 & 111 & 112 & 111 & 111 & 112 & 111 \\
\hline \multirow[t]{3}{*}{ SecularAuthorityTrust } & & & .559 & .434 & .526 & .692 & .748 & .760 & .485 & .404 \\
\hline & & & .000 & .000 & .000 & .000 & .000 & .000 & .000 & .000 \\
\hline & & & 111 & 112 & 111 & 112 & 111 & 111 & 112 & 111 \\
\hline \multirow[t]{3}{*}{ RA Investment } & & & & .781 & .944 & .572 & .529 & .586 & .848 & .774 \\
\hline & & & & .000 & .000 & .000 & .000 & .000 & .000 & .000 \\
\hline & & & & 111 & 111 & 111 & 110 & 110 & 111 & 111 \\
\hline \multirow[t]{3}{*}{ RA Expectation } & & & & & .944 & .353 & .523 & .455 & .853 & .747 \\
\hline & & & & & .000 & .000 & .000 & .000 & .000 & .000 \\
\hline & & & & & 111 & 112 & 111 & 111 & 112 & 111 \\
\hline \multirow[t]{3}{*}{ RA Trust } & & & & & & .490 & .558 & .552 & .901 & .806 \\
\hline & & & & & & .000 & .000 & .000 & .000 & .000 \\
\hline & & & & & & 111 & 110 & 110 & 111 & 111 \\
\hline \multirow[t]{3}{*}{ NA Investment } & & & & & & & .781 & .953 & .378 & .516 \\
\hline & & & & & & & .000 & .000 & .000 & .000 \\
\hline & & & & & & & 111 & 111 & 112 & 111 \\
\hline \multirow[t]{3}{*}{ NA Expectation } & & & & & & & & .933 & .449 & .532 \\
\hline & & & & & & & & .000 & .000 & .000 \\
\hline & & & & & & & & 111 & 111 & 110 \\
\hline \multirow[t]{3}{*}{ NA Trust } & & & & & & & & & .435 & .554 \\
\hline & & & & & & & & & .000 & .000 \\
\hline & & & & & & & & & 111 & 110 \\
\hline \multirow[t]{3}{*}{ RA in-group Trust } & & & & & & & & & & .501 \\
\hline & & & & & & & & & & .000 \\
\hline & & & & & & & & & & 111 \\
\hline
\end{tabular}

Fig. 2 Correlations table

country-specific religious landscapes of our four countries. A repeated measures ANOVA revealed a significant interaction between country and RA $F(3,107)=$ $6.022, p=.001$. Splitting by country (Fig. 4) reveals that Trust in RA in-group differs significantly from trust in RA out-group: US: $F(1,36)=40.089, p=.000, \mathrm{CZ}$ :

USA

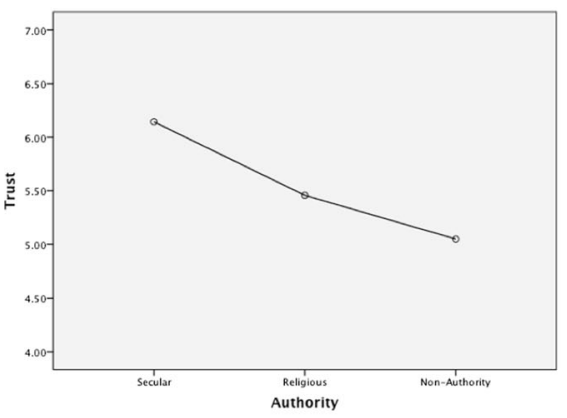

Mauritius

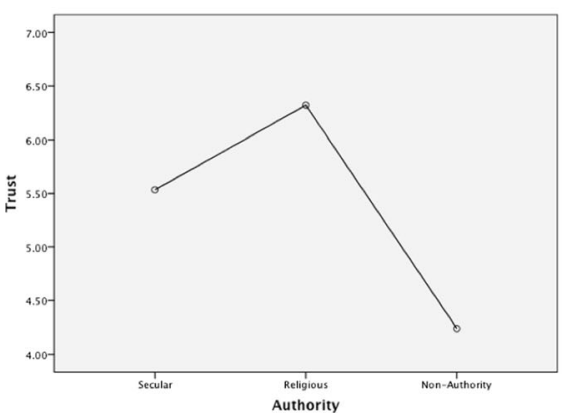

Czech Republic

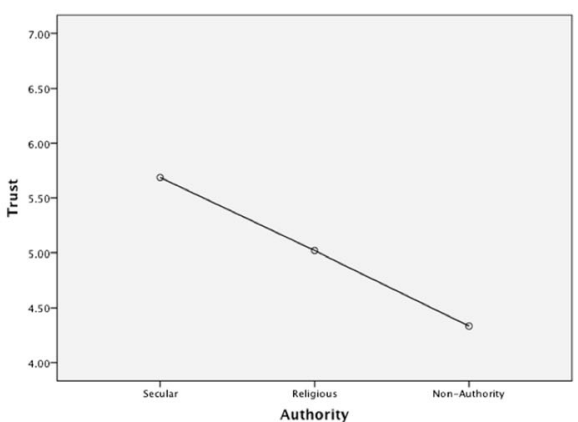

Denmark

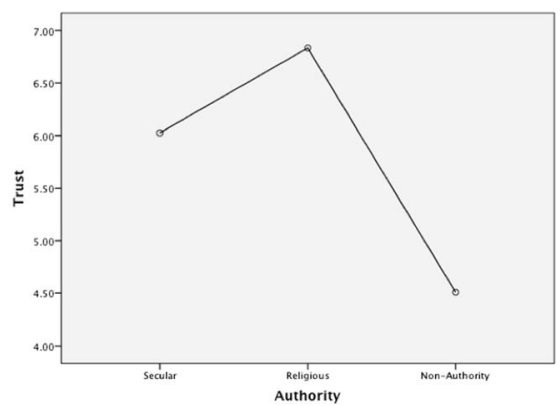

Fig. 3 Effects of authority on trust in each of the countries 

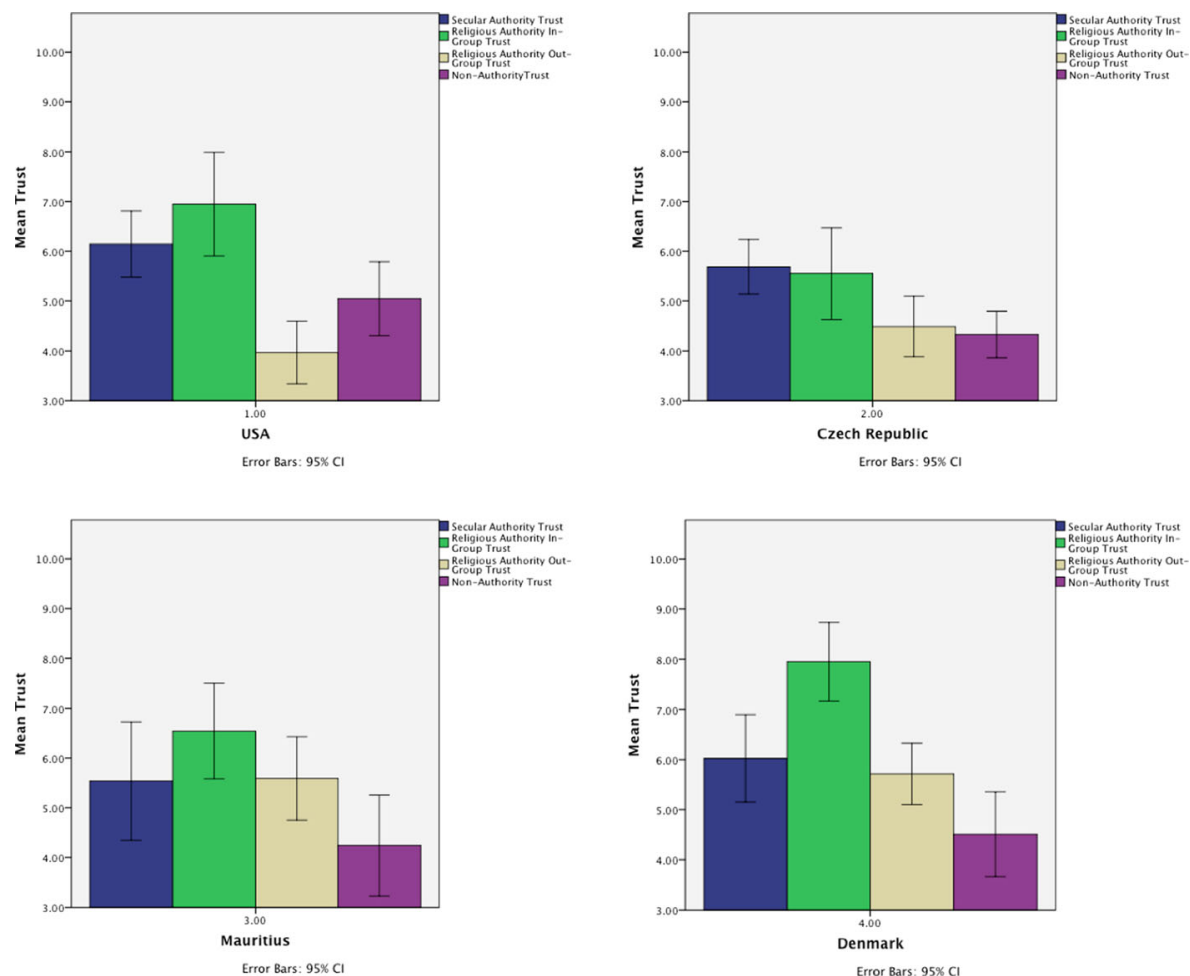

Fig. 4 Effects of religious authority (In-/out- group) on trust in each of the countries

$F(1,33)=12.103, p=.001, \mathrm{MU}: F(1,19)=4.638, p=.044$, and DK: $F(1,19)=34.517$, $p=.000$.

Based on the above results, we ran simple post-hoc tests, to explore how each country differs from the others in trust towards in-group vs. out-group RA: the US significantly differs from both the $\mathrm{CZ}(p=.000)$ and $\mathrm{MU}(p=.001)$ and the $\mathrm{CZ}$ marginally differs from DK $(p=.058)$.

Additionally, we added the split condition back into the model and ran a repeated measures ANOVA with 4 conditions (SA, RA in-group, RA out-group, NA): $F(9,318)=5.481, p=.000, \eta_{\mathrm{p}}{ }^{2}=.052$. Spiting by country results into: US: $F(3,108)=$ $3.372, p=.000, \eta_{\mathrm{p}}{ }^{2}=.311, \mathrm{CZ}: F(3,96)=9.235, p=.000, \eta_{\mathrm{p}}{ }^{2}=.170, \mathrm{MU}: F(3,57)=8.686$, $p=.000, \eta_{\mathrm{p}}{ }^{2}=.233$ and DK: $F(3,57)=33.114, p=000, \eta_{\mathrm{p}}{ }^{2}=.535$.

We did not find any significant effects in the self-report items (all $p>.111$ ).

\section{Discussion}

Cooperation between unrelated individuals inherently creates situations where personal and collective interests are at odds (for a working definition of cooperation: Mitkidis et al. 2013). In smaller-scale social realms, agents can decide to engage in or to withdraw from cooperation based on a firmer knowledge of other individual's character, personality and typical behaviors. They can acquire new strategic information about 
less well-known individuals on the cheap through grapevine or gossip. They can also generally calibrate their engagement in social exchange by relying on baselines and benchmarks extrapolated from prior histories of systematic or sustained interactions. Larger-scale social worlds afford much more complex situations of informal social exchange. In such societies, the agents whom one is likely to encounter are more likely to be more numerous, to be unknown, not to share the same social fabric and educational background, and not to occupy similar social and economic positions and roles. Anonymity, and the greater isolation from social retaliation that it permits, incentivizes selfish agents to steeply discount the future payoffs that cooperation might bring; to free-ride, to defect or to cheat. In such conditions, given the increased uncertainty about the chance of interacting with a genuinely cooperative individual, rational agents should desist from investing in informal social exchange until more information is acquired, a condition which is very often difficult to obtain systematically. Many regulatory apparatuses of human societies serve the essential function of limiting the risks associated with such situations of social dilemma, and the more developed the society, the more numerous and refined the regulatory devices become. However, informal social exchanges, by definition, are not channeled through such mechanisms. In modern societies, many informal interactions bring about situations where cooperation does not prevail, despite all parties knowing that by coordinating their investment to engage in a fruitful social exchange they would enhance their respective payoff.

If the assessment presented above - of the inherent problem of coordination between unrelated and unknown agents in situation of informal social exchange - is correct, then systemic and codependent forms of trust (category- and role-based), should increasingly play an essential role in large complex societies. People should increasingly, therefore, accept greater risk (i.e. invest in cooperative endeavor) than the available evidence would justify on the basis of information and knowledge about salient roles and positions in the societal and organizational structures. Our research has addressed the specific question of the impact of expertise and authority, as honest (read: hard-tofake) signals, on cooperation. Our experimental research relied on a TG design that allowed us to easily operationalize the evolutionarily bizarre but socially essential situation in which agents depend extensively on alien others. The design also lends itself to a straightforward manipulation of the amount and specificity of the social information conveyed. We provided one key measure of societal role - one's profession - and manipulated whether that profession was one of Secular Authority, Religious Authority, or was of No Authority.

In the four countries where we ran the experiment, the United States (US); the Czech Republic (CZ); Mauritius (MU); and Denmark (DK), we found differing significant interactions between types of authority and the measures of investment and expectation. The significant interactions are maintained even while the measures of investment and expectation are collapsed into an aggregate measure of Trust. Patterns of behavior, however, differ dramatically between countries, falling into two basic patterns. The US and the CZ behaved very similarly to each other, both with relatively high Trust for secular authorities, medium Trust for religious authorities, and low Trust for nonauthorities; while the DK and MU behaved in a different pattern, with medium Trust for secular authorities, high Trust for religious authorities, and low Trust for nonauthorities (see Fig. 3). 
In a recent poll released by the Win-Gallup International, the $\mathrm{CZ}$ is ranked in the top ten Atheist populations in the world (Gallup International Association 2012). Twenty percent declare being religious, $48 \%$, non-religious and $30 \%$, atheist. The profile of Trust in the CZ (Fig. 3) seems self-explanatory in light of this poll. At first sight, religious authorities seem to be granted less Trust than secular authorities. However, it is noteworthy that religious authorities are still trusted significantly more than nonauthorities, despite the affirmed atheism in the $\mathrm{CZ}$. This can probably be explained by the participant's knowledge of the inherent constraints that the social visibility of those positions imposes on such authorities. Behaving inappropriately, if detected, can have dramatic reputational consequences. An indication that this is indeed what boosts our CZ participant's Trust in religious authorities (if compared to non-authorities) can be detected in the consequence of splitting the Trust into commonly encountered religious authorities (e.g. Roman Catholic priests - in-group) vs. exotic religious authorities (e.g. Rabbis - out-group) (Fig. 4). Trust in in-group religious authorities aligns very neatly with Trust in secular authorities, while Trust in out-group authorities is significantly lower, more or less decreased to the level of Trust in non-authorities. The status of religious authorities belonging to one's group (even if one is a dedicated atheist) can be monitored more readily than the status of members of other cultural communities.

Trust in religious authorities in the US seems harder to explain given the general understanding that the country has moderate to high religiosity (Gallup International Association 2012). Religious authorities are only significantly more trusted than nonauthorities. Given the great geographic mobility of significant segments of the US population, that Trust in non-authorities be so low is understandable. But what about Trust in religious authorities? The above-mentioned Win-Gallup poll offers a line of reasoning. The US has experienced a precipitous drop in religiosity from $73 \%$ in 2005 to $60 \%$ in 2012, $13 \%$ in 6 years (Gallup International Association 2012). This could plausibly explain our findings of tepid Trust in religious authorities. But once again, as in the case of the CZ, splitting between Trusts in in-group vs. out-group religious authorities is revealing (Fig. 4). Trust in in-group religious authorities differs significantly from Trust in out-group religious authorities and is significantly higher than Trust in secular authorities. The same rationale as the one presented above for the $\mathrm{CZ}$ can be made here for the US. In-group religious authorities have to worry about the reputational impacts of their actions. US participants are generally more religiously involved than the $\mathrm{CZ}$ participants, which would partially explain the greater impact of the split between in-group and out-group. Distrust in out-group religious authorities is also driving the effect. Once isolated, Trust in out-group religious authorities is significantly lower than Trust in non-authorities. This is the single country where that effect can be observed (Fig. 4).

Both MU and DK place a premium on occupying a position that confers religious authority. Religious authorities are relatively more trusted than secular ones. When splitting between in-group and out-group, Trust in out-group religious authorities is maintained at the level of Trust for secular authorities (Fig. 4). We should remember that the two countries differ diametrically in their demographic homogeneity (i.e. Fractionalization Index) and Religious Diversity Index (Alesina et al. 2003; Pew Research Center 2014). Yet both countries behave similarly in trust allocation. The explanation for the observed patterns can be found in the particular ethnic, social, institutional and political structures of the two countries. 
MU has three major religious blocks: a Hindu majority (56.4\%) and two important minorities, Christian (25.3\%) and Muslim (16.7 \%) (Pew Research Center 2014). ${ }^{4}$ A paltry .6\% is considered religiously non-affiliated (Pew Research Center 2014). Religious memberships follow the existing ethnic demarcations (Indo-Mauritian, Creole, Muslims from Indian subcontinent immigration) (Carroll and Carroll 2000; Greig et al. 2011; Kasenally 2011). Despite decisive trends toward more centralization and national integration, ethnic affiliations to a great extent still define people's sphere of socialization and economic opportunities (Carroll and Carroll 2000). Religious groups and associations are often seen as primary sources of help and support (Greig et al. 2011). In such a context, it is not surprising that Mauritian participants significantly trust more religious authorities overall, and their in-group authorities more than the other categories. In Mauritius, religious authorities and associations seem to be an essential and salient part of the social world, far more so than secular authorities and associations. Religious associations and authorities are key in organizing the provisioning of social services, and thus are highly visible, and more likely to be exposed to opprobrium if they fail to live to collective expectations. (Kasenally 2011).

The general profile of DK is quite different from the Mauritian one. Eighty-three percent of the Danish population belongs (even if nominally) to the Danish Evangelical Lutheran Church denomination, the official religion of DK (Pew Research Center 2014). The Danish Evangelical Lutheran Church, its institutions and practices (e.g. marriage, funerals, issuance of birth certificates) are seamlessly woven into the social fabric, even though a vast majority of people do not consider themselves religious (Zuckerman 2008, 2009). Being ethnically Danish to a point still means that one is a nominal member of the Evangelical Lutheran Church (Zuckerman 2008). Once again, in such a social landscape, occupying the position of a religious dignitary comes with responsibility and duties and the concomitant reputational risk. The status, position and recognition of religious authorities rest heavily upon the support they find in their community, while secular authorities rely more strongly on the existence of formal institutions and systems of legitimation, assessment and enforcement.

A few final observations based on our experimental findings: For Trust in religious authorities, the US differs from the CZ only, and the latter, from MU and DK. The significantly lower Trust in religious authorities in the $\mathrm{CZ}$ drives the effects. We do not have an explanation for why that would be the case, though atheism might be part of the picture. But why are atheism and non-religiosity so prevalent in the $\mathrm{CZ}$ and not in the other surrounding societies? Many countries in Central Europe have had similar recent histories (i.e. communist control) but display very different religious profiles (see: Pew Research Center 2014). Part of the explanation might be found in the main authorities that lead the opposition to communism. In Poland, for instance, the church was heavily involved in the opposition, while in the $\mathrm{CZ}$ it was not. The matter needs to be investigated further. Finally, there is no significant difference for Trust in secular authorities, and in all four countries the effect of expertise and authority is evident: all authorities are significantly trusted more than non-authorities, as the increase in cooperation in the TG indicates. This seems to suggest that information about one's

\footnotetext{
${ }^{4}$ According to Statistics Mauritius (2012) the figures are: Hindu majority (49\%) and two important minorities, Christian (32\%) and Muslim (17\%).
} 
role in an organizational structure is indeed systematically used to make decisions in information-poor situations in modern social landscapes.

There are specific limitations in the study. They come from the complicated nature of a cross-cultural experiment. For privacy protection and IRB compliance, we did not collect certain pieces of information, such as our participant's religious or economic background. Our pools of participants (students in the CZ, MU and DK and state-fairgoers in the USA) may not be representative of the general population of our selected countries. and an additional limitation may be that our populations could be considered not-homogeneous (students in the CZ, MU and DK and state-fair population in the USA). Further studies are needed to identify, for example, possible order effects (such as contrast effects, where people would give authorities more after having been first asked how much they would give to non-authorities). Despite its limitations, our study adds to the small amount of existing empirical work examining the behavioral consequences of authority and expertise in informal social exchange.

Acknowledgments This research was funded by the Laboratory for the Experimental Research of Religion at Masaryk University (LEVYNA, CZ.1.07/2.3.00/20.0048), co-financed by the European Social Fund and the state budget of the Czech Republic; the "Technologies of the Mind" project at the Interactive Minds Centre at Aarhus University; the Velux Foundation; and the Center for Advanced Hindsight at Duke University. We thank Manesha Soneea and Martin Šmíra for assistance during the study. We also thank Jesper Sørensen, Kristoffer L. Nielbo, and Uffe Schjødt for their valuable suggestions in the early phases of the project, Sebastian Wallot for his comments on the text and the two anonymous reviewers for their excellent suggestions. Finally, we thank our informers in the four different countries for their help during the design of the study.

Author Contributions $\quad$ P. Mitkidis and P. Lienard designed the experiment. P. Mitkidis, D. Xygalatas, N. Buttrick and M. Porubanova collected the data. P. Mitkidis and N. Buttrick developed the stimuli. P. Mitkidis analyzed the data. P. Mitkidis, N. Buttrick, and P. Lienard interpreted the data. P. Mitkidis and P. Lienard wrote the article.

Conflict of Interest Authors declare no competing financial interest and approved the final version of the article for submission.

\section{References}

Aghion, P., \& Tirole, J. (1997). Formal and real authority in organizations. J Polit Econ, 105(1), 1-29.

Alesina, A., et al. (2003). Fractionalization. J Econ Growth, 8(2), 155-94.

Bacharach, M., Guerra, G., \& Zizzo, D. (2007). The self-fulfilling property of trust: an experimental study. Theor Decis, 63, 349-388.

Berg, J., Dickhaut, J., \& McCabe, K. (1995). Trust, reciprocity, and social history. Game Eco Behav, 10, 122142.

Butler, J. K. (1991). Toward understanding and measuring conditions of trust: evolution of a conditions of trust inventory. J Manag, 17, 643-663.

Carroll, B. W., \& Carroll, T. (2000). Trouble in paradise: ethnic conflict in mauritius. J Commonw Comp Polit, $38(2), 25-50$.

Charness, G., \& Dufwenberg, M. (2006). Promises and partnership. Econometrica, 74, 1579-1601.

Cohen, J. (1988). Statistical Power Analysis for the Behavioral Sciences (2nd ed.). Hillsdale: Erlbaum.

Cook, K., Hardin, R., \& Levi, M. (2005). Cooperation Without Trust? The Russell Sage Foundation Series On Trust. New York: Russel Sage Foundation.

Cox, J. (2004). How to identify trust and reciprocity. Game Econ Behav, 46, 260-281. 
Eckel, C., \& Wilson, R. (2004). Is trust a risky decision? J Econ Behav Organ, 55, 447-465.

Fukuyama, F. (1995). Trust: The social virtues and the creation of prosperity. London: Hamish Hamilton.

Gallup International Association, (2012). Global Index of Religiosity and Atheism, WIN-Gallup International.

Gambetta, D. (1988). Can we trust trust? In D. Gambetta (Ed.), Trust: Making and Breaking Cooperative Relations. New York: Blackwell.

Granovetter, M. (2005). The impact of social structure on economic outcomes. J Econ Perspect, 19(1), 33-50.

Greig, A., Turner, M., \& D'Arcy, P. (2011). The fragility of success: repositioning mauritian development in the twenty-first century. Island Stud J, 6(2), 157-178.

Gurtman, M. B. (1992). Trust, distrust, and inter- personal problems: a circumplex analysis. J Pers Soc Psychol, 62, 989-1002.

Hardin, R. (2002). Trust And Trustworthiness. The Russell Sage Foundation Series On Trust. New York: Russell Sage Foundation.

Houser, D., Schunk, D., \& Winter, J. (2010). Distinguishing trust from risk: an anatomy of the investment game. J Econ Behav Organ, 74, 72-81.

Johnson, N., \& Mislin, A. (2011). Trust games: a meta-analysis. J Econ Psychol, 32, 865-889.

Kasenally, R. (2011). Mauritius: paradise reconsidered. J Democr, 22(2), 160-169.

Kramer, R. M. (1999). Trust and distrust in organizations: emerging perspectives, enduring questions. Annu Rev Psychol, 50(1), 569-598.

Lane, C. (1998). Introduction. In C. Lane \& R. Bachman (Eds.), Theories and issues in the study of trust in Trust within and between organizations (pp. 1-30). Oxford: Oxford University Press.

Lewis, J. D., \& Weigert, A. (1985). Trust as a social reality. Soc Forces, 63, 967-85.

Luhman, N. (1979). Trust and power. New York: Wiley.

McCabe, K., Rigdon, M., \& Smith, V. (2003). Positive reciprocity and intentions in trust games. J Econ Behav Organ, 52, 267-275.

Mitkidis, P., Sørensen, J., Nielbo, K., Andersen, M., \& Lienard, P. (2013). Collective goal ascription increases cooperation in humans. PLoS One, 8(5), e64776.

Orbell, J., Dawes, R., \& Schwartz-Shea, P. (1994). Trust, social categories, and individuals: the case of gender. Motiv Emot, 18(2), 109-128.

Parsons, T. (1951). The social system. London: Routledge \& Kegan Paul.

Pew Research Center, April 2014. Global Religious Diversity: Half of the Most Religiously Diverse Countries are in Asia-Pacific Region.

Richards, N. (2007). Country Studies Series: Mauritius. Coexistence International at Brandeis University. Waltham: Brandeis University.

Rotter, J. B. (1971). Generalized expectancies for interpersonal trust. Am Psychol, 26, 443-52.

Rotter, J. B. (1980). Interpersonal trust, trust- worthiness, and gullibility. Am Psychol, 35, 1-7.

Sapienza, P., Toldra, A., Zingales, L. Understanding trust. (2007). No. w13387. National Bureau of Economic Research.

Schechter, L. (2007). Traditional trust measurement and the risk confound: an experiment in rural Paraguay. $J$ Econ Behav Organ, 62, 272-292.

Shamir, B. and Lapidot, Y. (2003). Trust in Organizational Superiors: Systemic and Collective Considerations, Organization Studies 2003 24: 463.

Simmel, G. (1950). The sociology of Georg Simmel, K. H. Wollff (ed.). New York: Free Press.

Sorrentino, R. M., Holmes, J. G., Hanna, S. E., \& Sharp, A. (1995). Uncertainty orientation and trust in close relationships: individual differences in cognitive styles. J Pers Soc Psychol, 68, 314-27.

Srebrnik, H. (2000). Can an ethnically-based civil society succeed? The case of Mauritius. J Contemp Afr Stud, 18(1), 7-20.

Statistics Mauritius. (2012). 2011 Housing and Population Census. Ministry of Finance and Economic Development: Port Louis.

The Economist, 2013. Democracy Index 2012, Democracy at a standstill. The Economist Intelligence Unit, March 14th, London, New York, Hong Kong, Geneva, The Economist Intelligence Unit Limited.

Weber, M. (1947). The Theory of Social and Economic Organization. New York: The Free Press.

Zucker, L. (1986). Production of trust: institutional sources of economic structure. Res Org Behav, 8, 53-111.

Zuckerman, P. (2008). Society Without God. New York: NYU Press.

Zuckerman, P. (2009). Why Are Danes and Swedes So Irreligious?. Nordic Journal of Religion and Society, vol. 22 , no.1. 